\title{
Analysis of physiological parameters in skin tumors by a scaleable Monte Carlo simulation
}

Alejandro Garcia-Uribe, Lihong V. Wang

Alejandro Garcia-Uribe, Lihong V. Wang, "Analysis of physiological parameters in skin tumors by a scaleable Monte Carlo simulation," Proc. SPIE 5695, Optical Interactions with Tissue and Cells XVI, (15 April 2005); doi: 10.1117/12.590308

SPIE. Event: SPIE BiOS, 2005, San Jose, CA, United States 


\title{
Analysis of Physiological Parameters in Skin Tumors by a Scaleable Monte Carlo Simulation
}

\author{
Alejandro Garcia-Uribe ${ }^{1}$, Lihong V. Wang ${ }^{2,1}$ \\ ${ }^{1}$ Department of Electrical Engineering, Texas A\&M University \\ 3128 TAMU, College Station, Texas 77843-3128 \\ ${ }^{2}$ Department of Biomedical Engineering, Texas A\&M University \\ 3120 TAMU, College Station, Texas 77843-3120
}

\begin{abstract}
This paper presents a study of the use of a single scaleable Monte Carlo simulation to estimate the physiological parameters of skin lesions from data collected in vivo using spectroscopic oblique-incidence reflectometry. Spatiospectral data from 101 cases are separated into two groups based on their melanocytic conditions. Group-1 consists of (a) cancerous basal cell carcinomas and squamous cell carcinomas and (b) benign actinic keratoses and seborrheic keratoses. Group-2 consists of (a) dysplastic nevi and (b) benign common nevi. Several physiological parameters are estimated, such as the size distribution of the optical scatterers, the relative index of refraction of the scatterers, the total volume concentration of the scatterers, the concentration of the total hemoglobin and the oxygen saturation, and the relative changes related to the values calculated from the neighboring healthy tissues. The most significant features are then combined into one feature. The results show that for both groups the combined feature is significantly different for the benign and cancerous cases than for the dysplastic cases. The ROC area was 0.9 and 0.86 for group- 1 and group- 2 , respectively.
\end{abstract}

Keywords: Oblique incidence reflectometry, Spectroscopy, Monte Carlo, Skin Cancer, Lesion classification, Physiological origin

\section{INTRODUCTION}

Skin cancer is the most common of all cancers in humans, and its incidence has increased dramatically in recent years. Malignant melanoma (MM) is the most serious type of skin cancer. Other types of skin cancer, such as basal cell carcinoma (BCC) and squamous cell carcinoma (SCC), are called non-melanoma cancers. In the United States alone, more than one million cases of non-melanoma skin cancer were diagnosed in 2003. ${ }^{1}$ Melanoma accounted for about 54,200 cases, and it is responsible for about 7,600 of the 9,800 deaths due to skin cancer reported in $2003 .{ }^{1}$ Oblique incidence reflectometry has an advantage over the the classically used normal incidence method because it gathers information specifically from the superficial layers of the skin. This is of particular importance since skin cancer is usually present in the top layers of the skin tissue, and the deeper layers only add to the background noise in the signal. ${ }^{2}$ Monte Carlo simulation was used to extract optical properties from measured diffuse reflectance. The principles of Monte Carlo simulation of photon transport have been thoroughly described by Wang et al. ${ }^{3}$ The Monte Carlo method is often used to solve the transport equation numerically, but it is too slow to be used repetitively in an inverse algorithm for deducing optical properties. Assuming a semi-infinite homogeneous media, ${ }^{4,5}$ a single scaleable Monte Carlo simulation can be used to deduce the optical properties. This scaleable simulation is possible because the Monte Carlo simulation results for the reference values of the index of refraction $n_{r}$, the anisotropy factor $g$, the absorption coefficients $\mu_{a}$, and the scattering coefficient $\mu_{s}$ can be used to calculate the desired quantities for all possible absorption coefficients $\mu_{a}$ by applying Beer's law. ${ }^{5}$ The results can be scaled for all scattering coefficients $\mu_{s}{ }^{\prime}$ while the refractive index $n_{r}$ and $g$ are constants because different $\mu_{s}$ values change only the distances between the interaction points on the photon paths. ${ }^{5}$

\section{SPECTRAL IMAGING SYSTEM}

Our oblique incidence diffuse reflectance spectroscopy (OIDRS) system is illustrated in Figure 1. A white light is coupled to a single optical fiber that delivers light to the sample to be irradiated. As indicated in Figure 1, the source 
fiber delivers the light while a linear array of 13 fibers $(200 \mu \mathrm{m}$ diameter low-OH optical fibers) collects the diffusely reflected light. The output of the connecting interface is placed at the object plane of the imaging spectrograph (Oriel MS257). With the probe placed normal to the surface of the sample, the source fiber is oriented at a 45 degree angle of incidence. The spectrograph generates the light spectrum and projects it onto a CCD camera (Princeton instrument Inc. 1530P) to form a spatio-spectral image as shown in Figure 2. In this figure the horizontal axis reflects the spatial location as seen by the fibers, and the vertical axis represents the spectral distribution of the light from each fiber.

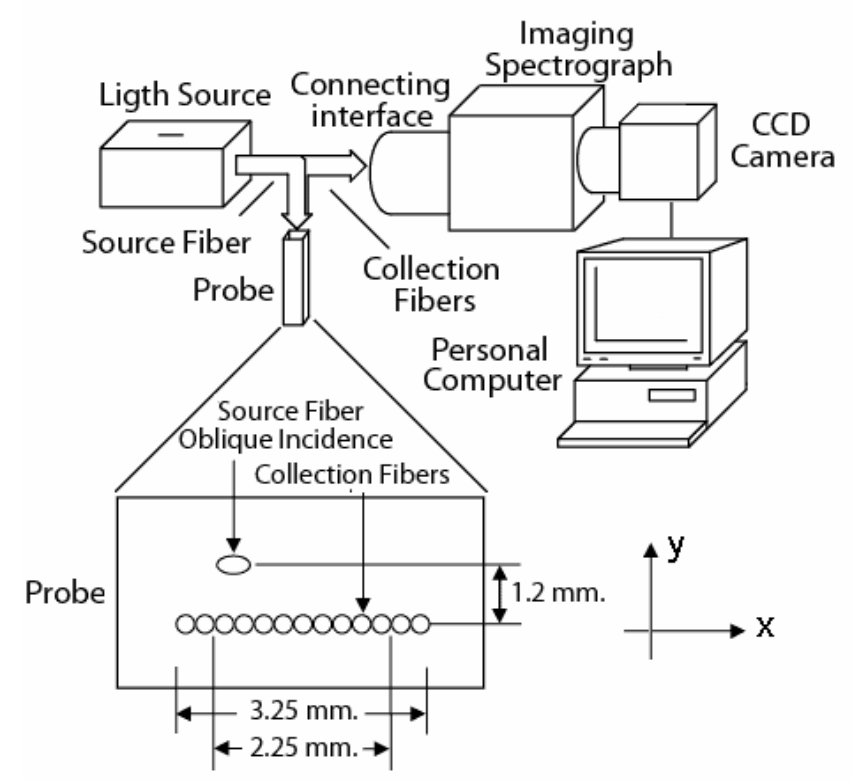

Figure 1. Diffuse Reflectance Spectroscopic Imaging System.

The image acquisition for our studies was performed at the University of Texas MD Anderson Cancer Center. The suspicious lesions were divided into two groups. Group 1 corresponded to the non-melanoma cases consisting of cancerous basal and squamous cell carcinomas and benign seborrhoeic keratosis. Group 2 consisted of pre-cancerous dysplastic nevi and benign common, compound and junctional nevi. These groups were chosen since in each group the benign cases resemble their respective cancerous cases making diagnosis difficult. ${ }^{6}$ For the group- 1 classifier, 304 spectral images were collected from 36 cases (13 cancerous and 23 benign), which consisted of 152 spectral images from the lesions and the same number of images from neighboring healthy tissues. For the group- 2 classifier, 522 spectral images from 65 cases (36 dysplasia and 29 benign) were collected, 261 images from the skin lesions and 182 images from neighboring healthy tissues.

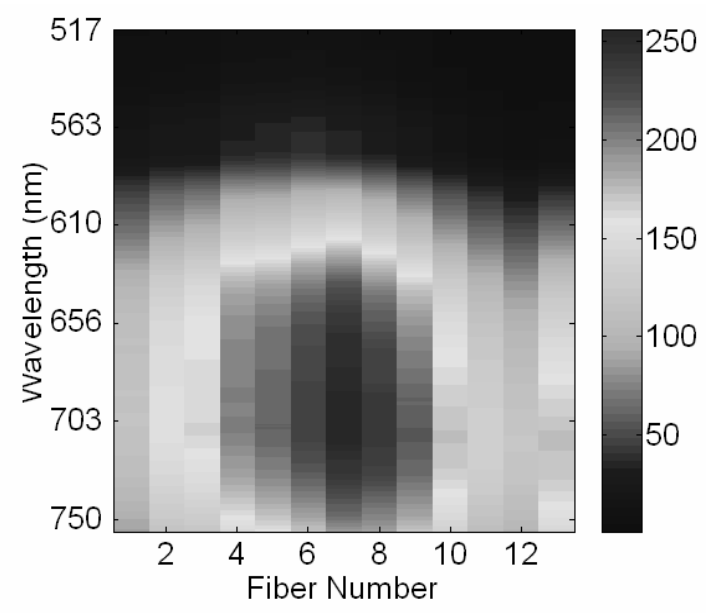

Figure 2. Sample spatio-spectral image. 


\section{SCALABLE MONTE-CARLO}

The algorithm is briefly summarized here. A single reference initial Monte Carlo simulation was run with a fixed anisotropy factor $g$ at 0.9 . The pre-calculated time-resolved diffuse reflectance $R_{r}(x, y, t)$ was computed for reference parameters $\mu_{a r}$ and $\mu_{s r}$ and saved in a computer file. The time-resolved diffuse reflectance $R(x, y, t)$ for any new parameters, $\mu_{a}$ and $\mu^{\prime}$, was calculated based on the following relationship:

$$
R(x, y, t)=\left(\frac{\mu_{s}}{\mu_{s r}}\right)^{3} R_{r}\left(x \frac{\mu_{s}}{\mu_{s r}}, y \frac{\mu_{s}}{\mu_{s r}}, t \frac{\mu_{s}}{\mu_{s r}}\right) \exp \left[-\left(\mu_{a}-\mu_{a r}\right) \frac{\mu_{s}}{\mu_{s r}} \frac{c}{n} t\right]
$$

where $c$ is the speed of light in a vacuum and $n$ is the index of refraction of the tissue. Since the perpendicular distance $y_{o}=1.2 \mathrm{~mm}$ between any collection fiber and the source fiber is constant, $R(x, t)$ was obtained by first scaling in the $\mathrm{y}$ direction. The corresponding steady-state diffuse reflectance was calculated by

$$
R(x)=\int_{0}^{\infty} R(x, t) d t
$$

The absorption spectra $\mu_{a}(\lambda)$ can be represented by ${ }^{7}$

$$
\mu_{a}(\lambda)=\varepsilon_{o x}(\lambda) C_{o x}+\varepsilon_{d e}(\lambda) C_{d e}+\varepsilon_{m e l}(\lambda) C_{m e l}+\mu_{a b g}
$$

where $\mu_{a}\left(\mathrm{~cm}^{-1}\right)$ is the absorption coefficient; $\lambda$ is the wavelength; $\varepsilon_{o x}(\lambda), \varepsilon_{d e}(\lambda)$, and $\varepsilon_{m e l}(\lambda)$ are the known extinction coefficients, $\left(\mathrm{cm}^{-1} \mathrm{mM}^{-1}\right)$ of oxy-hemoglobin, deoxy-hemoglobin, and melanin; $C_{o x}, C_{d e}$, and $C_{m e l}$ are the concentrations $(\mathrm{mM})$ of oxy-hemoglobin, deoxyhemoglobin, and melanin; and $\mu_{a b g}$ is the absorption coefficient caused by the local tissue components other than hemoglobins and melanin. The size distribution of the optical scatterers $f(\phi)$ can be calculated from the reduce scattering coefficient ${ }^{8} \mu_{s}{ }^{6}=\mu_{s}[1-g]$ using the following relationship:

$$
\mu^{\prime}(\lambda)=C_{s} \int_{0}^{\infty} \frac{3 Q_{s}(\phi, n, \lambda)[1-g(\phi, n, \lambda)]}{2 \phi} f(\phi) d \phi
$$

where $C_{s}$ is the total volume concentration of the scatterers; $Q_{s}()$ is the scattering efficiency; $\phi$ is the diameter of the scatterers, and $g()$ is the scattering anisotropy. $Q_{s}()$ and $g()$ were calculated using Mie Theory.

The Mitochondria, other cytoplasmic organelles and structures within the cell nuclei, are expected to be significant light scatterers. ${ }^{10,11}$ The average effective size of the scattering centers is assumed to correlate well with the size of the cell nuclei. Because our OIDR system provides diffuse reflectance for multiple wavelengths, we can fit the entire spatiospectral image for a spectra $\mu_{a}(\lambda)$ and $\mu_{s}(\lambda)$. To simplify the inverse problem, the scattering spectra $\mu_{s}(\lambda)$ is linearized and represented by the equation $\mu_{s}(\lambda) \approx m_{s} \lambda+b_{s}$ over a range of wavelength from $517 \mathrm{~nm}$ to $604 \mathrm{~nm}$. The fitted parameters that define $\mu_{a}(\lambda)$ are the concentrations of $C_{o x}, C_{d e}$, and $C_{m e l}$ directly. An example of the clinical data and the corresponding fitted image is shown in figure 3 . 


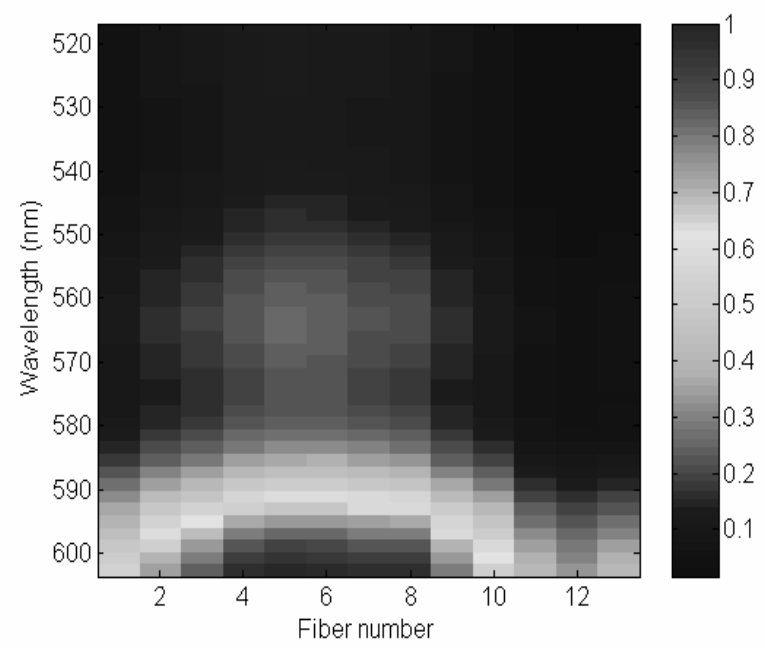

(a)

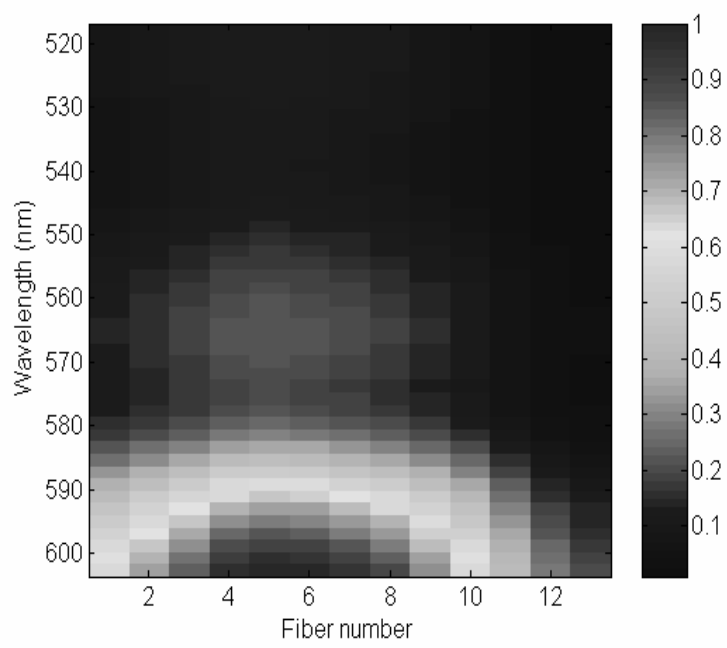

(b)

Figure 3. (a) Clinical spatio-spectral image. (b) Fitted spatio-spectral image.

The estimated linear $\mu_{s}(\lambda) \approx m_{s} \lambda+b_{s}$ was used later to calculate the size distribution of the optical scatters $f(\phi)$ using Eq. 4. $f(\phi)$ is assumed to be a Gaussian distribution $\mathrm{N}(\mu, \sigma)$. The oxygen saturation $\mathrm{SO}_{2}$ and the concentration of total hemoglobin $C_{H b}$ were computed based on the equations: $S O_{2}=C_{o x} /\left(C_{o x}+C_{d e}\right)$ and $C_{H b}=C_{o x}+C_{d e}$.

The concentrations of $C_{o x}, C_{d e}$, and $C_{m e l}$, their derived physiological parameters and the size distributions of $f(\phi)$ were estimated from the average spatio-spectral image for all skin lesions $(\mathrm{L})$ and their corresponding average image from neighboring healthy tissue $(\mathrm{H})$. The extracted values from the neighboring healthy tissues were used as a reference, or for normalization, to eliminate any background artifacts appearing in the lesion images. Figure 4 shows the normalized oxygen saturation $\mathrm{SO}_{2}$ and the mean and standard deviation of the size distributions of the optical scatters.

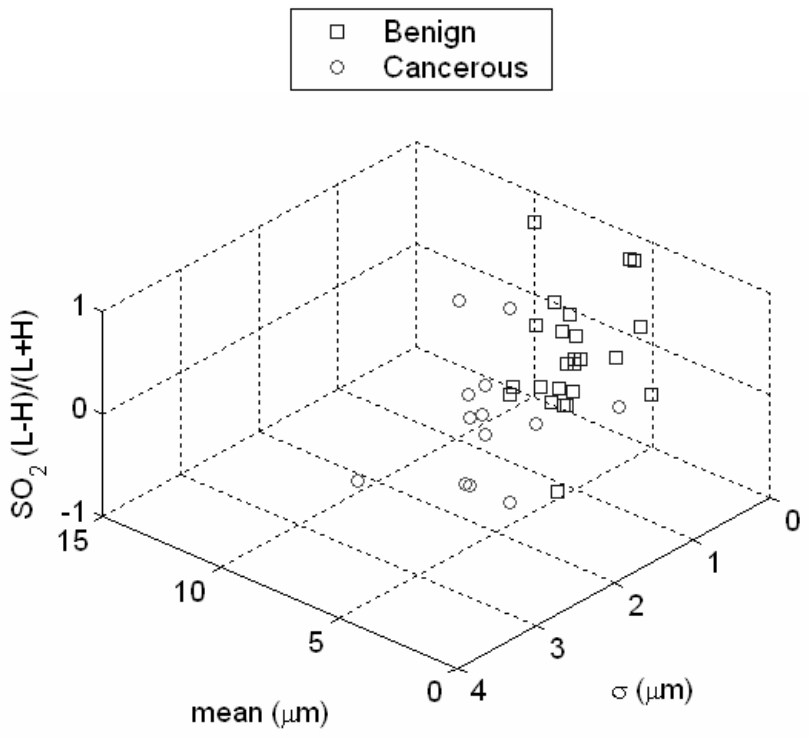

(a)

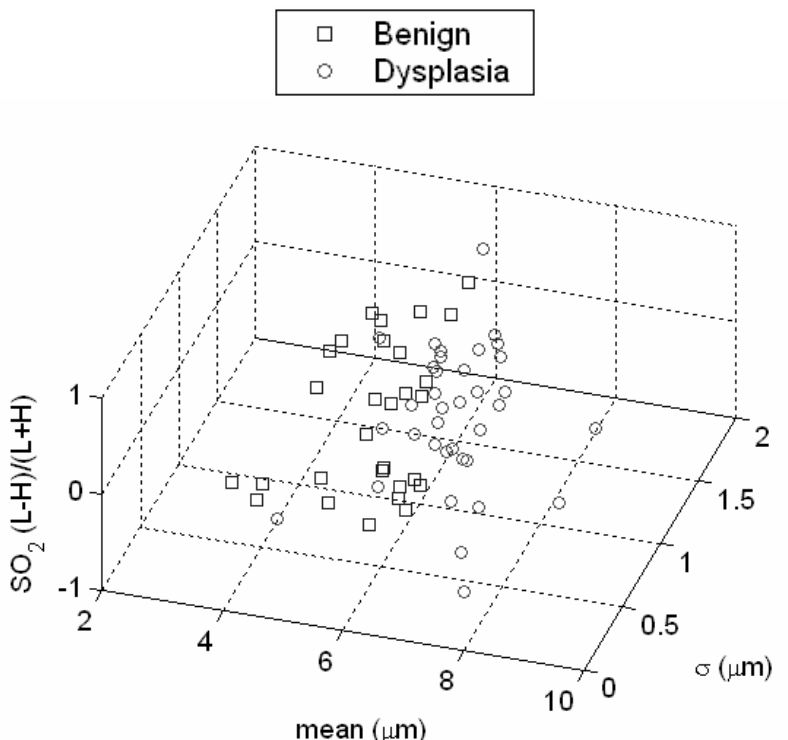

(b)

Figure 4. (a) Physiological parameters Group-1. (b) Physiological parameters Group-2 
These 3 physiological parameters for each case were combined into a single value. ${ }^{12}$ This final single value was used to calculate the receiver operating characteristic curve (ROC). The ROC curve describes the relationship between the true positives and the false positives for different threshold values on a scatter plot. The abscissa of the ROC curve is 1specificity, and its ordinate is sensitivity. Sensitivity indicates the fraction of correctly identified positive cases among all positive cases. The area under the ROC curve is a reflection of how effective the feature is at distinguishing between the classes. The ROC area is 0.9 and 0.86 for group- 1 and group-2, respectively. Figure 5 shows the ROC curve for group-1 and group-2, respectively.

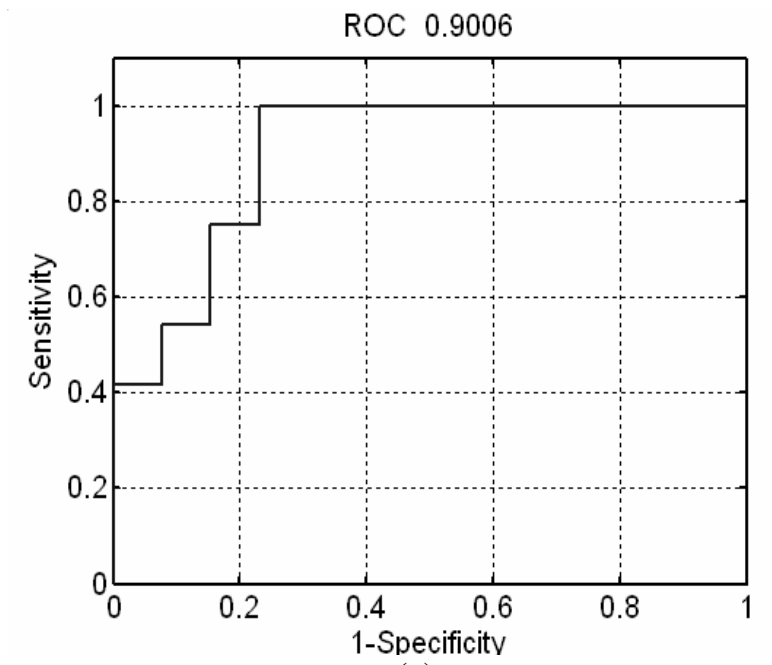

(a)

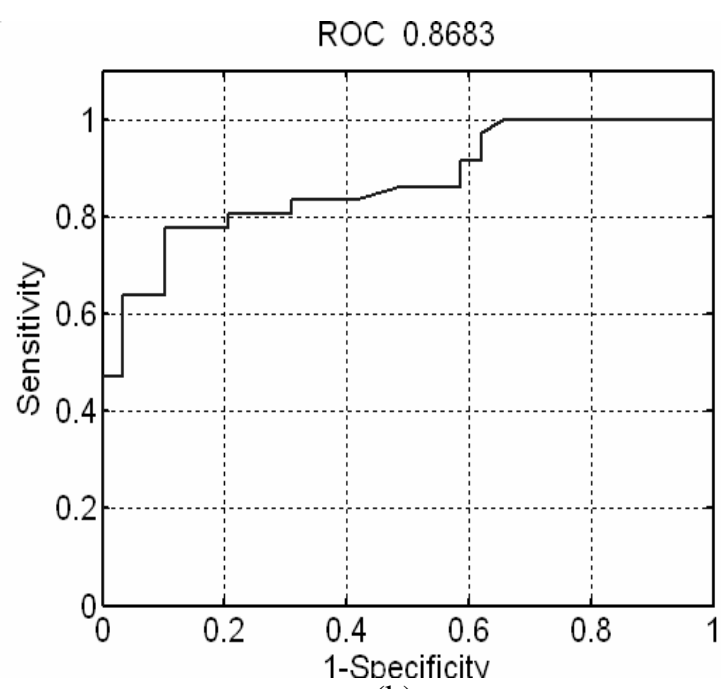

(b)

Figure 5. (a) ROC Group-1. (b) ROC Group-2

\section{CONCLUSIONS}

The results show that the expected value of the size distributions of the scatters $f(\phi)$ in the cancerous and dysplasia cases is larger than that in the benign cases. The cancerous lesions in group-1 have a lower average relative oxygen saturation $\mathrm{SO}_{2}$ than the benign lesions. Also dysplastic nevi cases present lower relative $\mathrm{SO}_{2}$ than common nevi. The lower oxygen saturation in cancerous and dysplastic nevi lesions can be related to abnormal blood supply and distribution and metabolic abnormalities.

\section{ACKNOWLEDGMENT}

This project is sponsored by NIH grant R01 CA106728. Wang's email is LWang@tamu.edu.

\section{REFERENCES}

1. American Cancer Society web site. http://www.cancer.org

2. G. Marquez, and L. H. Wang, "White Light Oblique Incidence Reflectometer for Measuring Absorption and Reduced Scattering Spectra of Tissue-like Turbid Media," Optics Express 1, 454-460, (1997).

3. L.-H. Wang, S. L. Jacques, and L.-Q. Zheng, "MCML - Monte Carlo modeling of photon transport in multi-layered tissues," Computer Methods and Programs in Biomedicine 47, 131-146, (1995).

4. R. Graaff, M. H. Koelink, F. F. M. de Mul, W. G. Zijlstr, A. C. M. Dassel, and J. G. Aarnoudse, "Condensed Monte Carlo simulations for the description of light transport," Appl. Opt. 32, 426-434 (1993). 
5. A. Kienle and M. S. Patterson, "Determination Of the Optical Properties Of Turbid Media From a Single Monte Carlo Simulation," Phys. Med. Biol. 41, 2221-2227 (1996).

6. G. Marquez, "Optical Biopsy for the Early Detection of Skin Cancer", Doctoral Dissertation, Texas A\&M University, December 2001.

7. H. Liu, D. A. Boas, Y. Zhang, A. G. Yodh, and B. Chance, "Determination of optical properties and blood oxygenation in tissue using continuous NIR light," Physics in Medicine \& Biology 40, 1983-93 (1995).

8. H. B. Jiang, J. Pierce, J. Kao, and E. Sevick-Muraca, "Measurement of particle-size distribution and volume fraction in concentrated suspensions with photon migration techniques," Appl. Opt. 36, 3310-3318 (1997).

9. H. C. van de Hulst, Light Scattering by Small Particles, Dover Publications, Inc., New York, (1981).

10. J. R. Mourant, James P. Freyer, Andreas H. Hielscher, Angelia A. Eick, Dan Shen, and Tamara M. Johnson, "Mechanisms of Light Scattering from Biological Cells Relevant to Noninvasive Optical-Tissue Diagnostics," Applied Optics 37, 3586-3593 (1998).

11. J. R. Mourant. Canpolat, C. Brocker, O. Esponda-Ramos, T. M. Johnson, A. Matanock, K. Stetter, and J. P. Freyer, "Light scattering from cells: the contribution of the nucleus and the effects of proliferative status," J. Biomed. Opt. 5, 131-137 (2000).

12. R. O. Duda, P .E. Hart, D. G. Stork, "Pattern Classification," New York: Wiley Interscience Publication, second edition, (2000). 